\title{
Learning curve of graft bench operation in living donor liver transplantation: a cumulative sum analysis
}

Jeong-Moo Lee, Kwangpyo Hong, Eui Soo Han, Sanggyun Suh, Su young Hong, Suk Kyun Hong, YoungRok Choi, Nam-Joon Yi, Kwang-Woong Lee, Kyung-Suk Suh

Department of Surgery-Hepatobiliary, Seoul National University Hospital, Seoul, Korea

Background: The middle hepatic vein (MHV) reconstruction is a critical issue for successful living donor liver transplantation. we analyzed the learning curve of MHV reconstruction and described the factors affecting the learning curve, and the postoperative outcomes.

Methods: Data from donors undergoing bench surgery between January 2019 to May 2020 retrospectively reviewed. To overcome operator-dependent bias, data from procedures performed by only a single surgeon (JML) were included. The learning curve was evaluated using the cumulative sum (CUSUM) method based on operative time.

Results: A total of 111 bench surgery were evaluated. The mean operative time was $64.0 \pm 15.8$ minutes, and the reconstructed MHV graft patency rate was $88.3 \%$ in recipient CT taken 7 days after liver transplantation. Portal vein stenosis occurred in three cases (2.7\%). Hepatic artery complications were four (3.6\%) and biliary complications were $18.1 \%$, and no graft failure occurred during the study period. Univariable analysis showed that portal vein variation, presence of more than two factors of contributing difficulty were associated with a significantly higher risk of prolonged operative time. These factors are also significantly associated with prolonged operative time in multivariable analysis.

Conclusions: At least 10 cases of learning curve are required for successful bench surgery in routine case of living donor liver transplantation. Multiple portal vein orifice is related with longer operative time and learning curve for the bench operation.

Corresponding author: Jeong-Moo Lee

E-mail:jmleetpl@gmail.com

(c) The Korean Society for Transplantation

This is an Open Access article distributed under the terms of the Creative Commons Attribution Non-Commercial License (http://creativecommons.org/licenses/by-nc/4.0/) which permits unrestricted non-commercial use, distribution, and reproduction in any medium, provided the original work is properly cited. 